17. Ho, L., et al. 2005. MALT1 and the API2-MALT1 fusion act between CD40 and IKK and confer NFkappa B-dependent proliferative advantage and resistance against FAS-induced cell death in B cells. Blood. 105:2891-2899.

18. Zhou, H., et al. 2004. Bcl10 activates the NF-kap$\mathrm{paB}$ pathway through ubiquitination of NEMO. Nature. 427:167-171.

19. Che, T., et al. 2004. MALT1/paracaspase is a signaling component downstream of CARMA1 and mediates T cell receptor-induced NF-kappaB activation. J. Biol. Chem. 279:15870-15876.

20. Lucas, P.C., et al. 2001. Bcl10 and MALT1, independent targets of chromosomal translocation in malt lymphoma, cooperate in a novel NF-kappa B signaling pathway. J. Biol. Chem. 276:19012-19019.

21. Zhou, H., Du, M.Q., and Dixit, V.M. 2005. Constitutive NF-kappaB activation by the $t(11 ; 18)(\mathrm{q} 21 ; \mathrm{q} 21)$ product in MALT lymphoma is linked to deregulated ubiquitin ligase activity. Cancer Cell. 7:425-431.
22. Hu, S., et al. 2006. cIAP2 is a ubiquitin protein ligase for BCL10 and is dysregulated in mucosaassociated lymphoid tissue lymphomas. J. Clin. Invest. 116:174-181. doi:10.1172/JCI25641.

23. Nakagawa, M., et al. 2005. MALT1 contains nuclear export signals and regulates cytoplasmic localization of BCL10. Blood. 106:4210-4216.

24. Ye, H., et al. 2005. MALT lymphoma with $\mathrm{t}(14 ; 18)(\mathrm{q} 32 ; \mathrm{q} 21) / \mathrm{IGH}-\mathrm{MALT} 1$ is characterized by strong cytoplasmic MALT1 and BCL10 expression. J. Pathol. 205:293-301.

25. Greiner, A., et al. 1994. Idiotype identity in a MALT-type lymphoma and B cells in Helicobacter pylori associated chronic gastritis. Lab. Invest. 70:572-578.

26. Hussell, T., Isaacson, P.G., Crabtree, J.E., Dogan, A., and Spencer, J. 1993. Immunoglobulin specificity of low grade B cell gastrointestinal lymphoma of mucosa-associated lymphoid tissue (MALT) type. Am. J. Pathol. 142:285-292.
27. Lenze, D., et al. 2005. Influence of antigen on the development of MALT-lymphoma. Blood. Online publication ahead of print.

28. Hussell, T., Isaacson, P.G., Crabtree, J.E., and Spencer, J. 1996. Helicobacter pylori-specific tumourinfiltrating $\mathrm{T}$ cells provide contact dependent help for the growth of malignant B cells in low-grade gastric lymphoma of mucosa-associated lymphoid tissue. J. Pathol. 178:122-127.

29. Dave, S.S., et al. 2004. Prediction of survival in follicular lymphoma based on molecular features of tumor-infiltrating immune cells. N. Engl. J. Med. 351:2159-2169.

30. de Jong, D. 2005. Molecular pathogenesis of follicular lymphoma: a cross talk of genetic and immunologic factors. J. Clin. Oncol. 23:6358-6363.

31. Rollinson, S., et al. 2003. Gastric marginal zone lymphoma is associated with polymorphisms in genes involved in inflammatory response and antioxidative capacity. Blood. 102:1007-1011.

\title{
Serum peptidome for cancer detection: spinning biologic trash into diagnostic gold
}

\author{
Lance A. Liotta and Emanuel F. Petricoin
}

Center for Applied Proteomics and Molecular Medicine, College of Arts and Sciences, George Mason University, Manassas, Virginia, USA.

\begin{abstract}
The low molecular weight region of the serum peptidome contains protein fragments derived from 2 sources: (a) high-abundance endogenous circulating proteins and (b) cell and tissue proteins. While some researchers have dismissed the serum peptidome as biological trash, recent work using mass spectrometry-based (MS-based) profiling has indicated that the peptidome may reflect biological events and contain diagnostic biomarkers. In this issue of the JCI, Villanueva et al. report on MS-based peptide profiling of serum samples from patients with advanced prostate, bladder, or breast cancer as well as from healthy controls (see the related article beginning on page 271). Surprisingly, the peptides identified as cancer-type-specific markers proved to be products of enzymatic breakdown generated after patient blood collection. The impact of these results on cancer biomarker discovery efforts is significant because it is widely believed that proteolysis occurring ex vivo should be suppressed because it destroys endogenous biomarkers. Villanueva et al. now suggest that this suppression may in fact be preventing biomarker generation.
\end{abstract}

\section{The illusion of a dry biomarker pipeline}

Despite the tremendous urgency to identify clinically useful biomarkers for early

Nonstandard abbreviations used: BRCA2, breast cancer 2, early onset; LMW, low molecular weight; MALDITOF, matrix-assisted laser desorption/ionization-timeof-flight; MS, mass spectrometric, mass spectrometry.

Conflict of interest: The authors have no personal conflicts, financial or otherwise. They have US government-assigned and -owned patent applications that cover aspects of serum biomarker discovery and amplification discussed in this manuscript. As former government employees, if the US government licenses these patents the authors are entitled to receive a share of the royalties.

Citation for this article: J. Clin. Invest. 116:26-30 (2006). doi:10.1172/JCI27467. disease detection, there have been only a few recent examples of such analytes that have had any real impact at the bedside $(1,2)$. Many scientists have pointed to what they perceive to be a dried-up blood-borne cancer biomarker pipeline for disease detection since recent searches for a single, cancer-specific marker have not proved fruitful. In response to this challenge, investigators in the field of proteomics have shifted their focus in an effort to utilize experimental methods such as mass spectrometry (MS), which does not require knowledge of a protein's amino acid sequence prior to effective detection of the analyte. These MS-based methods offer new approaches whereby signatures of multiple analytes measured simultaneously comprise the diagnostic classifier (3-10). MS analysis of blood proteome is proving facile at probing and profiling proteomic information that may encompass hundreds of candidate disease biomarkers without the need for a priori knowledge of their existence or relevance to disease states (4-10).

Within this field of research, interest continues to grow regarding a previously unexplored reservoir - the array of existing proteins in a patient's serum (coined as the serum proteome), particularly those of low molecular weight (LMW), as well as the metabolic products of these serum proteins (the serum peptidome, fragmentome, or degradome) $(11,12)$. Prior efforts in the search for serum and plasma protein biomarkers utilized gel-based separation technologies, which cannot readily separate and distinguish molecules of less than $10 \mathrm{kDa}$ in size. In contrast, MS is particularly well suited for the detection of molecules within the LMW range of analysis ( $<20 \mathrm{kDa})$. In recognition of this attribute, investigators began to use MS to explore the LMW component of the circulatory proteome in order to determine whether the LMW pool contained any disease-related biomarker candidates. This method was first applied to examine the sera of patients with ovarian cancer (4) and then later for other cancers (5-9) 


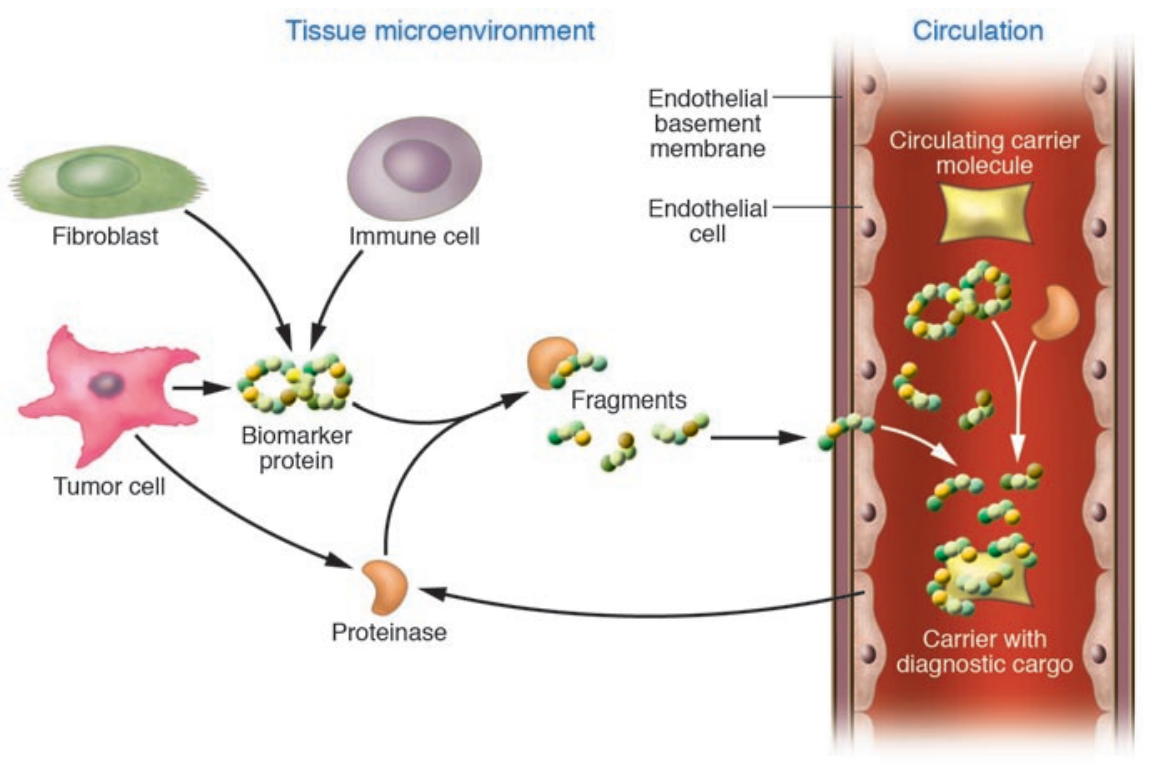

and nonneoplastic diseases (10). Early research studies revealed an apparent abundance of LMW proteins and peptides that potentially contain disease-specific information and showed that changes in the expression patterns of these molecules may be disease specific. However, these early signatures, derived from MS analysis of LMW serum proteins, comprised unidentified ions $(13,14)$. Subsequent efforts to sequence these diseasespecific ions revealed they were fragments derived from larger parent molecules that are normally too large to passively diffuse through the endothelium into the circulation and generally fall into 1 of 2 general categories: (a) fragments of endogenous, high-abundance proteins, such as transthyretin (15), and (b) fragments of low-abundance cellular and tissue proteins, such as breast cancer 2 , early onset (BRCA2) $(16,17)$. However, despite the potential richness of information contained within the serum peptidome, there remained cause for concern. Once sequenced, some putative disease marker ions were identified as fragments of highly abundant blood components found in healthy individuals. This led researchers to question the disease specificity of this repertoire of protein fragments (13).

\section{The serum peptidome: \\ from trash to treasure}

While some scientists have dismissed the LMW serum peptidome as noise, biological trash, or nonspecific epiphenomena $(13,14)$ too small to be biologically rel- evant, others have proposed that just the opposite is the case, that the LMW serum peptidome may contain a rich, untapped source of disease-specific diagnostic information (11). In this issue of the JCI, Villanueva et al. (18) provide evidence to this effect by utilizing MS-based serum peptidome profiling in order to identify qualitative and quantitative patterns or signatures that can indicate the presence or absence of specific types of cancer. The authors employed an automated peptide extraction technique utilizing magnetic, reverse-phase beads for analyte capture from subject sera coupled with matrix-assisted laser desorption/ionization-time-of-flight (MALDI-TOF) MS to generate a peptide signature that could classify patients with advanced prostate, breast, or bladder cancer and differentiate them from healthy controls. While these investigators did not employ noncancer inflammatory disease controls within their study, the results support the robustness of their disease-specific peptide signature since the set of marker peptides enabled highly accurate cancer class prediction for an independent validation set of prostate cancer samples.

\section{Cancer-specific peptidome fingerprints}

Sequence identification of the 61 marker peptide peaks that provided the greatest degree of cancer class separation as determined by statistical significance revealed that most of the cancer-type-specific biomarker fragments were generated in

\section{Figure 1}

Proteinases generate biomarker fragments. Circulating protein fragments generated in the diseased tissue microenviroment may serve as diagnostic protein markers. Proteolytic cascades within the tissue (a product of the interacting cellular ecology such as stromalepithelial interactions), immune cell MHC presentation, or apoptosis generate protein fragments that passively diffuse into the circulation. Shed LMW peptides are protected from kidney-mediated clearance by sequestration on abundant resident blood proteins such as albumin. According to the results presented by Villanueva et al. (18) in this issue of the $\mathrm{JCl}$, diagnostic protein fragments can also be generated ex vivo by circulating enzymes derived from the diseased tissue microenvironment acting on exogenously derived peptides produced by serum collection methodology (see Figure 10 in ref. 18).

patient serum by enzymatic cleavage at previously known endoprotease cleavage sites after the blood sample was collected from the patient (18). Consequently, Villanueva et al. propose that the LMW biomarkers that they found in this study are not expressed directly by the diseased tissue but are in fact generated ex vivo by proteinase-mediated enzymatic cleavage as part of the coagulation and complement activation pathways. The authors explain that fragments of endogenous blood proteins generated ex vivo serve as a substrate pool for disease-specific proteinases that arise from the tumor itself or within the tumor microenvironment. The specific substrates cleaved by the proteinases are themselves degradation products of the clotting cascade. The authors hypothesize that the cancer-type-specific signatures they detect within the LMW portion of the serum peptidome are an indirect snapshot of the enzyme activity of tumor cells. Therefore, in the authors' view, the resultant peptide signatures are composed of what could be considered as surrogate markers for the detection and classification of certain types of cancer.

\section{Serum as a superior biomarker source}

The conclusions that may be drawn from the Villanueva et al. study (18) have potentially significant implications for the field of biomarker research and commercial clinical diagnostics. The authors state that it appears that a large part of the human serum peptidome, as detected by their 
bead-mediated analyte capture/MADLITOF MS approach, is produced ex vivo by degradation of endogenous substrates by endogenous proteinases. Since the diagnostic signatures are produced by circulating, disease-specific proteinases that act on their precursor peptide substrates after the blood has been removed from the patient, proteolytic degradation occurring after serum harvesting and after blood clotting is necessary for the production of at least 1 class of LMW biomarkers and should not be suppressed by the addition of proteinase inhibitors. This recommendation is exactly the opposite of that of many scientists who advocate the immediate addition of protease inhibitors during the blood collection process to specifically inhibit protease activity believed to contaminate the biomarker archive. The relative merit of serum versus plasma as the diagnostic fluid of choice is hotly debated in the world of diagnostic biomarkers. The results reported here by Villanueva et al. suggest that, in contrast to serum collection, plasma collection (which suppresses the clotting cascade) will mask the presence of the disease-associated proteinases that ultimately act on the exogenous fragment pool and produce the MALDI-TOF diagnostic signatures. Consequently, the authors suggest that serum is inherently superior to plasma as a source of diagnostic information contained within the peptidome.

\section{Carrier protein-bound peptidome}

While Villanueva et al. (18) provide evidence to demonstrate one method by which the LMW peptidome is created ex vivo, there is a growing body of evidence that circulating blood already contains an abundance of protein fragments apparently derived from cells and tissues that are produced in vivo. Previous work by Tirumalai et al. (12) and most recently by Lowenthal et al. (16) has revealed a vast and diverse source of LMW and lowabundance fragments of cellular proteins that are not cleavage products of resident serum proteins. This view of the LMW peptidome is one in which low-abundance peptide biomarkers produced from specific and ongoing tumorigenic processes such as apoptosis, tumor-stromal interaction, vascularization, immune cell infiltration, and antigenic processing exist in a sequestered state, complexed to

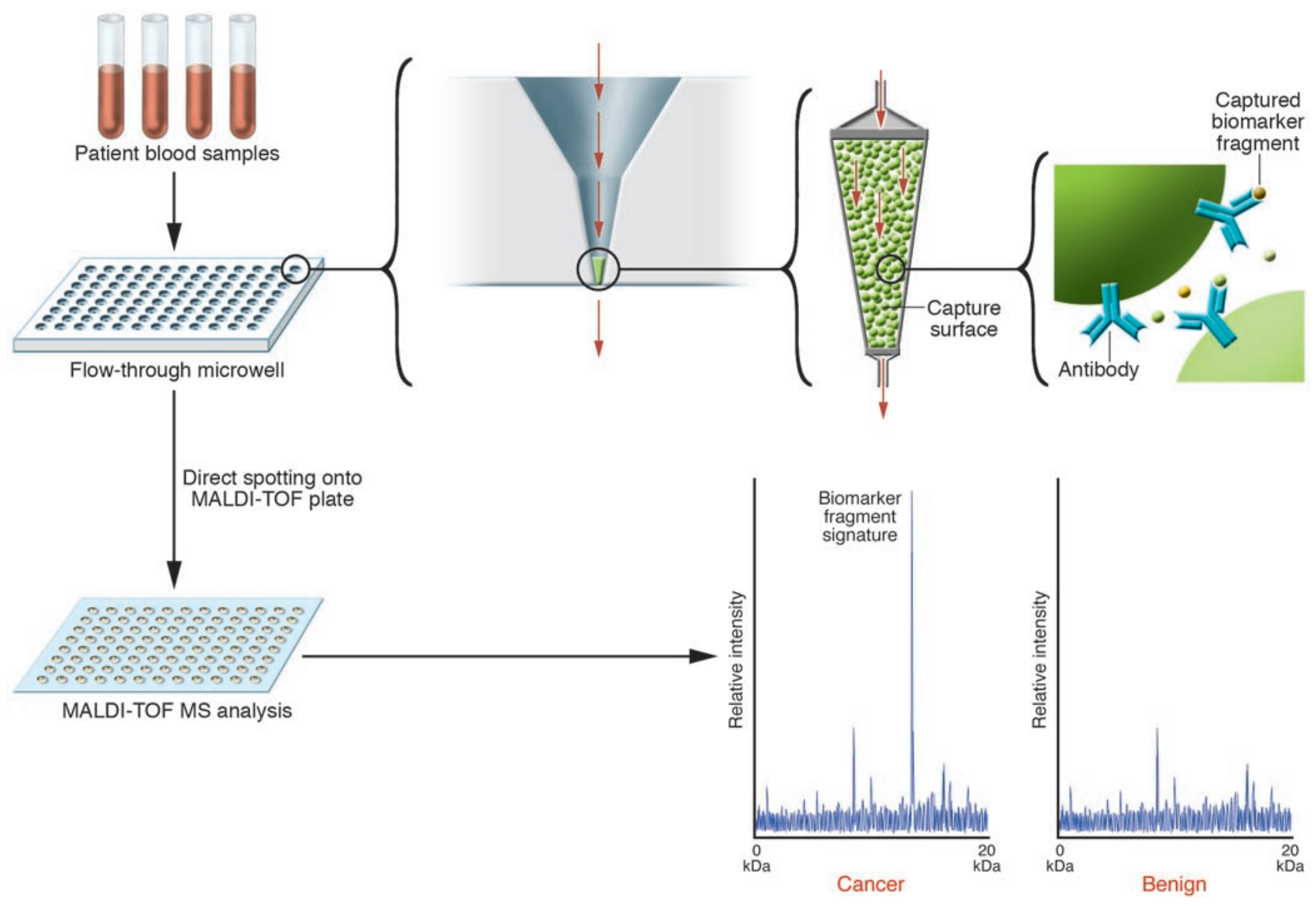

Figure 2

Immuno-MS provides a means for rapidly determining the specific size and identity of each member of a panel of peptide marker fragments present within patient sera. As part of a high-throughput assay performed in clinical diagnostic laboratories, patient serum would be applied to a multiplexed plate of microcolumns filled with antibody-immobilized beads. Each microcolumn captures both the parental and fragment isoforms of the candidate marker since they both contain the antibody recognition site. The captured population of analytes, including the fragment(s) with potential for disease detection and/or discrimination, are eluted and analyzed directly by MALDI-TOF MS. The presence of the specific peptide biomarker at its precise mass/charge ratio $(\mathrm{m} / \mathrm{z})$ would be used as a diagnostic test result. This analysis could be performed rapidly by simple software that determines if the ion peak is present or absent at the predefined $\mathrm{m} / \mathrm{z}$ location. 
highly abundant resident blood proteins such as albumin $(12,16,19)$ (Figure 1). The concentration of any LMW analyte in the circulation is completely dependant on its production and clearance rates. Given the ability of the glomeruli to efficiently and effectively remove molecules smaller than approximately $50 \mathrm{kDa}$, any LMW molecule generated in vivo would be cleared rather quickly, thereby reducing the concentration of the analyte at the time the blood is drawn to potentially undetectable levels. Sequestration of peptides by resident blood carrier proteins with long half-lives, such as albumin, protects the peptides from clearance. Two recent studies offer a first glimpse into the diagnostic potential of the LMW, carrier protein-bound biomarker pool. Lopez et al. report MALDI-TOF fingerprinting of serum for Alzheimer disease detection (20). They harvested albuminbound LMW protein fragments as the direct input for their MS ion fingerprint analyses. Alzheimer disease-associated fingerprints found in a training set were validated using an independent test set. The approach of Lopez et al. (20) was distinct from that used by Villeneuva et al. (18). A second study investigated the identities of LMW, albumin-bound fragments obtained from the sera of ovarian cancer patients (16). The authors identified a large number of ovarian cancerassociated peptides that were different from the peptides found in high-risk, disease-free subjects. A subset of peptides was found only in the samples from patients with stage 1 ovarian cancer. Sequencing of these peptides indicated that they were fragments of low-abundance molecules such as BRCA2, tyrosine kinases, other signaling molecules, and intracellular scaffolding proteins. Thus, the endogenous circulating peptidome may be potentially redefined as a subset of the blood interactome - protein analytes that exist in the circulation as protein-protein complexes. Identification of the components of the LMW circulatory peptidome by MS sequencing provides a list of specific analytes that is independent of the measurement technology (e.g., MALDI-TOF). Fingerprints of MS ions can therefore be replaced with panels of named protein biomarkers. Regardless of whether the peptidome is derived from the tissue/microenvironment in vivo or ex vivo, the implications for the diagnostics arena are enormous.

\section{Immuno-MS: the diagnostic platform of the future?}

Conventional immunoassay platforms cannot measure panels of analyte fragments. This is because immunoassays, by their very definition, rely on antibodybased capture and detection methods. An antibody-based assay cannot distinguish the parent molecule from its cleaved fragments (the latter of which could possess the greatest diagnostic potential) since the antibody recognizes its cognate epitope in both the parent and fragment molecules. Thus, the future of fragment-based diagnostics will require the invention and adoption of wholly new technologies that read out both the identity and the exact size of the molecule. Immuno-MS is 1 example of how this could be achieved (Figure 2). With this approach, a microaffinity antibody column, perhaps in a multiplexed microwell format, would first be used to capture the parent protein along with any of its fragment isoforms that contain the antibody recognition site. Next, the captured fragments would be eluted from the antibody column directly into a mass spectrometer (such as a MALDI-TOF MS). MS analysis of the eluted peptides would provide an extremely accurate mass determination of the entire population of captured peptides. Thus, in only 2 steps, a panel of peptide fragments derived from a known parent molecule could be rapidly tabulated. Future rounds of investigation into the nature and diagnostic potential of the serum peptidome will likely uncover many more surprises. Nevertheless, based on results to date, we now expect that the diagnostic potential of the LMW serum peptidome will likely be dependent on the examination of the following: (a) low-abundance, tissue-derived peptides, many of which avoid clearance by binding to carrier proteins; (b) size- and cleavage-specific fingerprints of fragments derived from cellular parent molecules; and (c) now, based on the findings of Villanueva et al. (18), additional signatures of cleavage products produced ex vivo after blood clotting during serum collection. Far from drying up, the LMW bloodborne biomarker pipeline is surging with potential. Nevertheless, this potential will never be realized unless commonly adopted and specific blood collection protocols are implemented across clinical and research laboratories, serum and plasma reference sets are developed and standardized, instrumentation for measuring panels of specific fragments are proven to be both sensitive and reproducible, and extensive validation of the diagnostic utility of these biomarkers is examined in clinical trials conducted in accordance with regulatory guidelines developed by the College of American Pathologists and Clinical Laboratory Improvement Amendments.

Address correspondence to: Lance A. Liotta or Emanuel F. Petricoin, Center for Applied Proteomics and Molecular Medicine, College of Arts and Sciences, George Mason University, 10900 University Boulevard, MS 4E3, Room 181A, Manassas, Virginia 20110, USA. Phone: (703) 993-9444; Fax: (703) 993-4288; E-mail: 1liotta@gmu.edu (L.A. Liotta). Phone: (703) 993-8646; Fax: (703) 993-4288; E-mail: epetrico@gmu.edu (E.F. Petricoin).

1. Anderson, N.L., and Anderson, N.G. 2002. The human plasma proteome: history, character, and diagnostic prospects. Mol. Cell. Proteomics. 1:845-867.

2. Petricoin, E.F., Fishman, D.A., Conrads, T.P., Veenstra, T.D., and Liotta, L.A. 2004. Lessons from Kitty Hawk: from feasibility to routine clinical use for the field of proteomic pattern diagnostics. Proteomics. 4:2357-2360

3. Skates, S.J., et al. 2004. Preoperative sensitivity and specificity for early-stage ovarian cancer when combining cancer antigen CA-125II, CA 15-3, CA 72-4, and macrophage colony-stimulating factor using mixtures of multivariate normal distributions. J. Clin. Oncol. 22:4059-4066.

4. Petricoin, E.F., et al. 2002. Use of proteomic patterns in serum to identify ovarian cancer. Lancet. 359:572-577.

5. Adam, B.L., et al. 2002. Serum protein fingerprinting coupled with a pattern-matching algorithm distinguishes prostate cancer from benign prostate hyperplasia and healthy men. Cancer Res. 62:3609-3614.

6. Li, J., Zhang, Z., Rosenzweig, J., Wang, Y.Y., and Chan, D.W. 2002. Proteomics and bioinformatics approaches for identification of serum biomarkers to detect breast cancer. Clin. Chem. 48:1296-1304.

7. Villanueva, J., et al. 2004. Serum peptide profiling by magnetic particle-assisted, automated sample processing and MALDI-TOF mass spectrometry. Anal. Chem. 76:1560-1570.

8. Hingorani, S.R., et al. 2003. Preinvasive and invasive ductal pancreatic cancer and its early detection in the mouse. Cancer Cell. 4:437-450.

9. Petricoin, E.F., 3rd, et al. 2002. Serum proteomic patterns for detection of prostate cancer. J. Natl. Cancer Inst. 94:1576-1578.

10. Stone, J.H., et al. 2005. Wegener's Granulomatosis Etanercept Trial Research Group. A serum proteomic approach to gauging the state of remission in Wegener's granulomatosis. Arthritis Rheum. 52:902-910.

11. Liotta, L.A., Ferrari, M., and Petricoin, E. 2003. Clinical proteomics: written in blood. Nature. 425:905.

12. Tirumalai, R.S., et al. 2003. Characterization of the low molecular weight human serum proteome. Mol. Cell. Proteomics. 2:1096-1103.

13. Diamandis, E.P. 2003. Point: proteomic patterns 
in biological fluids: do they represent the future of cancer diagnostics? Clin. Chem. 49:1272-1275.

14. Sorace, J.M., and Zhan, M. 2003. A data review and re-assessment of ovarian cancer serum proteomic profiling. BMC Bioinformatics. 4:24

15. Zhang, Z., et al. 2004. Three biomarkers identified from serum proteomic analysis for the detection of early stage ovarian cancer. Cancer Res. 64:5882-5890.
16. Lowenthal, M.S., et al. 2005. Analysis of albuminassociated peptides and proteins from ovarian cancer patients. Clin. Chem. 51:1933-1945.

17. Liotta, L.A., et al. 2005. Importance of communication between producers and consumers of publicly available experimental data. J. Natl. Cancer Inst. 97:310-314.

18. Villanueva, J., et al. 2006. Differential exoprotease activities confer tumor-specific serum peptidome patterns. J. Clin. Invest. 116:271-284. doi:10.1172/ JCI26022.

19. Zhou, M., et al. 2004. An investigation into the human serum "interactome". Electrophoresis. 25:1289-1298.

20. Lopez, M.F., et al. 2005. High-resolution serum proteomic profiling of Alzheimer disease samples reveals disease-specific, carrier-protein-bound mass signatures. Clin. Chem. 51:1946-1954.

\title{
Is the small heat shock protein $\alpha B$-crystallin an oncogene?
}

\author{
Sofia K. Gruvberger-Saal ${ }^{1}$ and Ramon Parsons ${ }^{2}$
}

1Department of Oncology, Lund University Hospital, Lund, Sweden. 'Departments of Pathology and Medicine, Institute for Cancer Genetics, Herbert Irving Comprehensive Cancer Center, Columbia University College of Physicians and Surgeons, New York, New York, USA.

In the last 5 years, global gene expression profiling has allowed for the subclassification of the heterogeneous disease of breast cancer into new subgroups with prognostic significance. However, for most subgroups, the nature of the contributions of individual genes to the clinical phenotypes remains largely unknown. In this issue of the JCI, Moyano and colleagues further examine the oncogenic potential of the small heat shock protein $\alpha$-basic-crystallin, commonly expressed in tumors of the basal-like breast cancer subtype associated with poor prognosis, and show that it is an oncogenic protein in the breast (see the related article beginning on page 261).

\section{Small heat shock proteins}

$\alpha$-Basic-crystallin ( $\alpha$ B-crystallin) is a member of the mammalian small heat shock protein (sHsp) superfamily, whose 10 -protein membership is defined by the presence of a conserved, approximately 90 -aa region (termed the $\alpha$-crystallin core) with divergent amino- and carboxyterminal domains (1-3). The sHsps function as cytoprotective molecular chaperones, preventing stress-induced aggregation of denaturing proteins as well as keeping aggregation-prone proteins in reservoirs of nonnative refoldable intermediates by holding proteins within large, soluble, multimeric structures. sHsps also appear to have a structural role; for example, $\alpha B$-crystallin together with $\alpha$-Acidic-crystallin comprise as much as $40 \%$ of the cytoplasmic proteins in lens cells of the eye and are thought to play an essential role in maintaining its

Nonstandard abbreviations used: $\alpha B$-crystallin, $\alpha$-basic-crystallin; CK, cytokeratin; ER, estrogen receptor; ErbB2, erythroblastic leukemia viral oncogene homolog 2; IHC, immunohistochemistry; MEK, MAPK kinase; sHsp, small heat shock protein.

Conflict of interest: The authors have declared that no conflict of interest exists.

Citation for this article: J. Clin. Invest. 116:30-32 (2006). doi:10.1172/JCI27462. transparency. However, in other tissues in the human body the $2 \alpha$-crystallins are expressed constitutively at much lower levels. $\alpha B$-Crystallin shares a close homology with another sHsp, Hsp27, and both of these proteins are activated in response to stresses such as heat shock, radiation, oxidative stress, and exposure to anticancer drugs. Moreover, both proteins have been shown to have antiapoptotic functions by interfering with the activity of various apoptotic proteins (2). Indeed, the literature suggests that $\alpha \mathrm{B}$-crystallin is important in the pathology of cancer, as overexpression of $\alpha \mathrm{B}$-crystallin has been observed in gliomas (4), renal carcinomas (5), breast carcinomas $(6,7)$, and ductal carcinoma in situ compared with matched normal breast tissue (8). Until now, the importance of $\alpha \mathrm{B}$-crystallin in cancer has been vaguely attributed only to its antiapoptotic functions.

\section{$\alpha B-C r y s t a l l i n$ as an oncoprotein}

In this issue of the JCI, Moyano and colleagues demonstrate properties of $\alpha \mathrm{B}$ crystallin not previously described to our knowledge that define it as a potential oncogenic protein (9). Using various cellculture systems, including reconstituted 3D basement membrane cultures, they report that constitutive overexpression of $\alpha \mathrm{B}$-crystallin in 2 human mammary epithelial cell lines, MCF-10A and MCF-12A, induced neoplastic-like changes such as EGF- and anchorage-independent growth, loss of polarity, disorganized acinar structure, increased proliferation, diminished apoptosis, and increased migration and invasion (Figure 1). These morphological changes were reliant on $\alpha \mathrm{B}$-crystallin overexpression, since retroviral RNA interference-mediated silencing of $\alpha B$-crystallin expression suppressed the abnormal phenotype. Moreover, although this transformation induced the expression and phosphorylation of ERK1/2, AKT, and p38, the authors show that the neoplastic changes were dependent on the ERK/MAPK pathway, as inhibition of only this pathway by highly specific MAPK kinase (MEK) inhibitors completely negated transformation. Transformation also appears to be dependent on the phosphorylation state of $\alpha B$ crystallin, as a pseudophosphorylation mutant of $\alpha \mathrm{B}$-crystallin, which mimics stress-induced phosphorylation, did not confer neoplastic changes. Furthermore, the authors demonstrate that $\alpha \mathrm{B}$-crystallin meets one of the "gold standards" for classification as an oncogenic protein: human mammary epithelial cells overexpressing WT $\alpha \mathrm{B}$-crystallin were shown to form invasive carcinomas in nude mice. In contrast, constitutive overexpression of the recognized oncogenes H-RasV12, cyclin D1, and erythroblastic leukemia viral oncogene homolog 2 (ErbB2) in these cells does not induce tumors in nude mice (10-12), suggesting that $\alpha \mathrm{B}$-crystallin is a more potent oncoprotein in this model. Finally, the authors demonstrate the clin- 